\title{
Local Volatility Pricing Models for Long-dated FX Derivatives
}

\author{
Griselda Deelstra and Grégory Rayée \\ Department of Mathematics, Université Libre de Bruxelles, \\ Boulevard du Triomphe, CP 210, Brussels 1050, Belgium, \\ e-mail: griselda.deelstra@ulb.ac.be \\ grayee@ulb.ac.be
}

April 4, 2012

\begin{abstract}
We study the local volatility function in the Foreign Exchange market where both domestic and foreign interest rates are stochastic. This model is suitable to price long-dated FX derivatives. We derive the local volatility function and obtain several results that can be used for the calibration of this local volatility on the FX option's market. Then, we study an extension to obtain a more general volatility model and propose a calibration method for the local volatility associated to this model.
\end{abstract}

Keywords : Local volatility; Stochastic volatility; Foreign Exchange; Stochastic interest rates; Calibration.

\section{Introduction}

Recent years, the long-dated FX option's market has grown considerably. Currently most traded and liquid long-dated FX Hybrid products are Power-Reverse Dual-Currency swaps (PRDC) (see for example (Piterbarg, 2006]) as well as vanilla or exotic long-dated products such as barrier options. While for short-dated options (less than 1 year), assuming constant interest rates does not lead to significant mispricing, for long-dated options the effect of interest rate volatility becomes increasingly pronounced with increasing maturity and can become as important as that of the FX spot volatility. Most of the dealers are using a three-factor pricing model for long-dated FX products (see Piterbarg, 2006, Sippel and Ohkoshi, 2002 ) where the FX spot is locally governed by a geometric Brownian motion, while each of the domestic and foreign interest rates follows a Hull-White one factor Gaussian model Hull and White, 1993. Using such a model does not allow the volatility smile/skew effect encountered in the FX market to be taken into account, and is therefore not appropriate to price and hedge long-dated FX products.

Different methods exist to incorporate smile/skew effects in the three-factor pricing model. In the literature, one can find different approaches which consist either of using a local volatility for the FX spot or a stochastic volatility and/or jump. There are many processes that can be used for the stochastic volatility and their choices will generally depend on their tractability and solvability. All 
these models should be calibrated over the market before being used for pricing. The calibration is normally based on calculating prices of liquid products for different strikes and maturity and the parameters of the model are adjusted until these prices match sufficiently with the market. However, in most cases it is difficult to derive analytical formulae, and consequently the calibration procedure often remains approximative or computationally demanding. Andreasen suggested in Andreasen, 2006 a stochastic volatility approach by combining a Heston [Heston, 1993 stochastic volatility model with independent stochastic interest rates. He has derived closed-form Fourier expressions for vanilla options which are useful for calibration on the vanilla market smile/skew. He has used an indirect approach in the form of a volatility displacement parameter to correlate the independent interest rates with the spot FX rate. In Antonov et al., 2008, Antonov et al. have underlined the problem that using non-trivial correlations destroys the affine structure and exact solvability. By using the technique of Markovian Projection, they have derived approximation formulae for the calibration of FX options in a three-factor model coupled with a Heston stochastic volatility under a full correlation structure. In van Haastrecht et al., 2009, van Haastrecht et al. have obtained the exact pricing of FX options under the three-factor model coupled with a Schöbel and Zhu Schöbel and Zhu, 1999] stochastic volatility and the full correlation structure. Their model could cover Poisson type jump with a trivial extension. Recently, van Haastrecht and Pelsser considered in van Haastrecht and Pelsser. 2009 the pricing of FX options under Schöbel and Zhu and also the Heston stochastic volatility with a multi-factor Gaussian interest rates and a full correlation structure. More precisely, they derived the characteristic functions required for the Fourier-based pricing methods under Schöbel and Zhu stochastic volatility. Unfortunately, they did not obtain a closed-form expression for the characteristic functions under the Heston stochastic volatility with a full correlation structure. However, they presented a calibration method based on the characteristic functions in the special uncorrelated case by using a projection of the general model onto the uncorrelated case, or by using it as a control variate for the general model. Using martingale methods and Fourier inversion techniques, Ahlip Ahlip, 2008 has derived an analytical formula for the price of a European call on the spot FX rate in the case of a stochastic volatility model with stochastic interest rates where domestic and foreign interest rates are modelled by Ornstein-Uhlenbeck processes and the instantaneous volatility follows a mean-reverting Ornstein-Uhlenbeck process correlated with the spot FX rate. Finally, L.A. Grzelak and C.W. Oosterlee Grzelak and Oosterlee, 2010] have derived semi-closed form approximations for the forward characteristic function in a foreign exchange model of Heston-type, in which the domestic and foreign interest rates are generated by the short-rate process of Hull-White and have extended the framework by modeling the interest rate by a stochastic volatility displaced-diffusion Libor Market Model.

A local volatility framework was explored by Piterbarg in Piterbarg, 2006 where the volatility of the spot FX rate was a function of both the time and the spot itself. He has derived an approximative formula for the local volatility which allows for a fast calibration of the model on vanillas. The calibration essentially captures the "slope" of the implied volatility surface but does not exactly fit its convexity.

Local volatility models, introduced in 1994 by Dupire Dupire, 1994 and Derman and Kani Derman and Kani, 1994] in the settings of equity, have the benefit over a stochastic volatility model that they are Markovian in only one factor since the local volatility is a deterministic function of both the FX spot and time. It avoids the problem of working in incomplete markets in comparison with stochastic volatility models and is therefore more appropriate for hedging strategies. Local volatil- 
ity models also have the advantage to be calibrated on the complete implied volatility surface, and consequently local volatility models usually capture more precisely the surface of implied volatilities than stochastic volatility models. However, a local volatility model has the drawback that it predicts unrealistic dynamics for the stock volatility since the volatilities observed in the market are really stochastic, capable of rising without a movement in spot FX prices. In [Bossens et al., 2010], the authors compare short-dated barrier option market prices with the corresponding prices derived from either a Dupire local volatility or a Heston stochastic volatility model both calibrated on the vanilla smile/skew. It appears from that study that in a simplified world where exotic option prices are derived either from Dupire local volatility or from Heston stochastic volatility dynamics, a FX market characterized by a mild skew (USDCHF) exhibits mainly a stochastic volatility behavior, and that FX markets characterized by a dominantly skewed implied volatility (USDJPY) exhibit a stronger local volatility component. This observation also underlines that calibrating a stochastic model to the vanilla market is by no means a guarantee that exotic options will be priced correctly Schoutens et al. 2004], as the vanilla market carries no information about the smile dynamics. The market dynamics could be better approximated by a hybrid volatility model that contains both stochastic volatility dynamics and local volatility ones. This approach has to our knowledge never been studied in a stochastic interest rates framework but gives positive results for short dated options when interest rates are assumed to be constant (see for example [Lipton, 2002, Lipton and McGhee, 2002, Madan et al., 2007, Tavella et al., 2006]). In the constant interest rates case, once the local volatility surface is available, the new mixed volatility can be computed by multiplying this local volatility with a ratio of integrals that depend on the joint density of the FX spot and the stochastic volatility. This density can be determined by numerically solving the associated two-dimensional Kolmogorov forward PDE.

The study of the local volatility and its calibration in a three-factor model with local volatility can therefore be motivated by hedging arguments but is also considerably useful for the calibration of hybrid volatility models. In this paper we derive the local volatility function in a three-factor model with local volatility where we have three sources of randomness: the FX spot with a local volatility and the domestic/foreign interest rates.

In a one-factor Gaussian model, the local volatility surface is generally built by using the Dupire's formula where partial derivatives of call options with respect to strikes and maturities are calculated by finite differences and where the real implied volatility surface is an interpolation of a finite set of market call prices. In a three-factor framework with local volatility, the expression of the local volatility becomes more complicated as it also depends on a particularly complicated expectation where no closed form expression exists and it is not directly related to European call prices or other liquid products. Its calculation can obviously be done by numerical integration methods or Monte Carlo simulations. To enable realizations of the numerical integrations you need the forward probability distribution of the spot FX rate and the domestic and foreign interest rates up to maturity which is a solution of a three-dimensional forward PDE.

An alternative approach is to calibrate the local volatility from stochastic volatility models by establishing links between local and stochastic volatility. Extracting the local volatility surface from a stochastic volatility model rather than by using the market implied volatility surface presents several advantages. First, the market implied volatility surface can in practice only be an interpolation of a finite set of available market data. As a consequence a local volatility surface built from an approximative implied volatility surface is often unstable. Stochastic volatility models can be calibrated by using 
fast algorithms like Fast Fourier Transforms (FFT) (see for example Ahlip, 2008, Andreasen, 2006. van Haastrecht et al., 2009]) and the local volatility surface extracted from the calibrated stochastic volatility model is really smooth. In this paper we will present some mimicking properties that links the three-factor model with a local volatility to the same model with a stochastic volatility rather than a local volatility. These properties will allow us to obtain explicit expressions to construct the local volatility surface.

Finally, we derive a link between the three-factor model with local volatility and a hybrid volatility model where the volatility of the spot FX rate mixes a stochastic volatility with a local volatility. Knowing the local volatility function associated to the three-factor model with local volatility, we propose a calibration method for the local volatility in the four-factor hybrid volatility model.

Our objective is to expose theoretical results about the local volatility function and its calibration. Numerical implementation of the model to the long-dated FX options market will be studied in a subsequent paper.

This paper is organized as follows: We begin by defining the three-factor model with local volatility in section 2. Then, in section 3 we derive the local volatility expression for this model. First, we derive from the three-dimensional Fokker-Plank equation for the forward probability density function, a "simpler" one-dimensional PDE. This PDE is used in the derivation of the local volatility function by differentiating European call price expressions with respect to the strike and the maturity. Section 4 is devoted to the calibration of this local volatility function. In section 4.1, we present two numerical approaches based on respectively Monte Carlo simulations and PDE numerical resolution. In section 4.2 we obtain a link between the local volatility function derived in a three-factor framework and the one coming from the simple one-factor Gaussian model. Next, in section 4.3, we derive a link between the three-factor model with a stochastic volatility for the spot FX rate and the one where the spot FX rate volatility is a local volatility. This link provides a relationship between our local volatility function and future instantaneous spot FX rate volatilities. Finally, in section 5 , we study an extension of the three-factor model with local volatility. We derive a link between the local volatility function associated to the three-factor model and the local volatility function present in the four-factor hybrid volatility model and propose a calibration procedure for the local volatility function associated to this four-factor hybrid volatility model. Conclusions are presented in section 6 .

\section{The three-factor pricing model with local volatility}

In this paper we consider the three-factor model where the spot FX rate volatility is a deterministic function of both the time and the FX spot itself. This function is known as 'local volatility'. In this model, the spot FX rate $S$ is governed by the following dynamics

$$
d S(t)=\left(r_{d}(t)-r_{f}(t)\right) S(t) d t+\sigma(t, S(t)) S(t) d W_{S}^{D R N}(t)
$$

where the volatility of the spot FX rate is a local volatility $\sigma(t, S(t))$ and where domestic and foreign interest rates denoted by $r_{d}$ and $r_{f}$ respectively follow a Hull-White one factor Gaussian model Hull and White, 1993 defined by the Ornstein-Uhlenbeck processes 


$$
\left\{\begin{array}{l}
d r_{d}(t)=\left[\theta_{d}(t)-\alpha_{d}(t) r_{d}(t)\right] d t+\sigma_{d}(t) d W_{d}^{D R N}(t), \\
d r_{f}(t)=\left[\theta_{f}(t)-\alpha_{f}(t) r_{f}(t)-\rho_{f S} \sigma_{f}(t) \sigma(t, S(t))\right] d t+\sigma_{f}(t) d W_{f}^{D R N}(t),
\end{array}\right.
$$

where $\theta_{d}(t), \alpha_{d}(t), \sigma_{d}(t), \theta_{f}(t), \alpha_{f}(t), \sigma_{f}(t)$ are deterministic functions of time. Equations (1), (2) and (3) are expressed in the domestic risk-neutral measure (DRN).

Foreign and domestic zero-coupon bonds defined by $P_{i}(t, T)=\mathbf{E}^{Q_{i}}\left[e^{-\int_{t}^{T} r_{i}(s) d s} \mid \mathcal{F}_{t}\right], i=d, f$ satisfy the evolution

$$
\left\{\begin{array}{l}
d P_{d}(t, T)=r_{d}(t) P_{d}(t, T) d t-\sigma_{d}(t, T) P_{d}(t, T) d W_{d}^{D R N}(t), \\
d P_{f}(t, T)=\left[r_{f}(t)+\rho_{f S} \sigma_{f}(t, T) \sigma(t, S(t))\right] P_{f}(t, T) d t-\sigma_{f}(t, T) P_{f}(t, T) d W_{f}^{D R N}(t),
\end{array}\right.
$$

where their volatility structures are given by

$$
\sigma_{i}(t, T)=\sigma_{i}(t) \int_{t}^{T} e^{-\int_{t}^{s} \alpha_{i}(u) d u} d s, \quad i=d, f
$$

More details about stochastic interest rates in the Hull-White one factor Gaussian model and explicit expression for the price of zero-coupon bonds under this framework can be found in Brigo and Mercurio, 2006.

Finally, in this paper we assume that $\left(W_{S}^{D R N}(t), W_{d}^{D R N}(t), W_{f}^{D R N}(t)\right)$ is a Brownian motion under the domestic risk-neutral measure $Q_{d}$ with correlation matrix

$$
\left(\begin{array}{ccc}
1 & \rho_{S d} & \rho_{S f} \\
\rho_{S d} & 1 & \rho_{d f} \\
\rho_{S f} & \rho_{d f} & 1
\end{array}\right)
$$

\section{The local volatility function}

In this section we derive the expression of the local volatility function by using the same technique as Dupire Dupire, 1994] and Derman and Kani Derman and Kani, 1994] which consists in differentiating European call price expressions with respect to the strike and the maturity.

\subsection{Forward PDE}

Consider a world where both the spot FX rate as well as the domestic and the foreign interest rates are stochastic. Under the assumption of absence of arbitrage opportunities, and working under the $t$-forward measure $Q_{t}$ (where the domestic zero-coupon bond is the numeraire), the present value $V\left(S(0), r_{d}(0), r_{f}(0), t=0\right)$ of a derivative that pays off $V\left(S(t), r_{d}(t), r_{f}(t), t\right)$ at time $t$ is given by

$$
\begin{aligned}
V\left(S(0), r_{d}(0), r_{f}(0), t=0\right) & =P_{d}(0, t) \mathbf{E}^{Q_{t}}\left[V\left(S(t), r_{d}(t), r_{f}(t), t\right)\right] \\
& =P_{d}(0, t) \iiint V(x, y, z, t) \phi_{F}(x, y, z, t) d x d y d z
\end{aligned}
$$


where $\phi_{F}(x, y, z, t)$ corresponds to the $t$-forward probability density.

Following the same approach as in 'Equity Hybrid Derivatives' Overhaus et al., 2006 (readapting the method in the context of FX market), we can get the following Fokker-Plank equation for $\phi_{F}(x, y, z, t)$

$$
\begin{aligned}
0= & \frac{\partial \phi_{F}}{\partial t}+\left(r_{d}(t)-f_{d}(0, t)\right) \phi_{F}+\frac{\partial\left[\left(r_{d}(t)-r_{f}(t)\right) S(t) \phi_{F}\right]}{\partial x}+\frac{\partial\left[\left(\theta_{d}(t)-\alpha_{d}(t) r_{d}(t)\right) \phi_{F}\right]}{\partial y} \\
& +\frac{\partial\left[\left(\theta_{f}(t)-\alpha_{f}(t) r_{f}(t)-\rho_{f S} \sigma_{f}(t) \sigma(t, S(t))\right) \phi_{F}\right]}{\partial z}-\frac{1}{2} \frac{\partial^{2}\left[\sigma^{2}(t, S(t)) S^{2}(t) \phi_{F}\right]}{\partial x^{2}}-\frac{1}{2} \frac{\partial^{2}\left[\sigma_{d}^{2}(t) \phi_{F}\right]}{\partial y^{2}} \\
& -\frac{1}{2} \frac{\partial^{2}\left[\sigma_{f}^{2}(t) \phi_{F}\right]}{\partial z^{2}}-\frac{\partial^{2}\left[\sigma(t, S(t)) S(t) \sigma_{d}(t) \rho_{S d} \phi_{F}\right]}{\partial x \partial y}-\frac{\partial^{2}\left[\sigma(t, S(t)) S(t) \sigma_{f}(t) \rho_{S f} \phi_{F}\right]}{\partial x \partial z}-\frac{\partial^{2}\left[\sigma_{d}(t) \sigma_{f}(t) \rho_{d f} \phi_{F}\right]}{\partial y \partial z} .
\end{aligned}
$$

Equation (6) is a forward PDE since it is solved forward in time with the initial condition at time $t=0$ given by $\phi_{F}(x, y, z, t)=\delta\left(x-x_{0}, y-y_{0}, z-z_{0}\right)$, where $\delta$ is the Dirac delta function and $x_{0}, y_{0}$ and $z_{0}$ correspond to the values at time $t=0$ of the spot FX rate, the domestic and foreign interest rates respectively. This initial condition means that at time $t=0$ we are sure that the spot FX rate $S(0)$ equals $x_{0}$, the domestic interest rate $r_{d}(0)$ equals $y_{0}$ and the foreign interest rate $r_{f}(0)$ equals $z_{0}$.

From equation (6) we will derive a "simpler" one-dimensional PDE only involving the dependence in $x$ of the forward density function. This equation will be useful in the derivation of the local volatility expression in section 3.2 .

Let us denote by $q_{F}(x, z, t)$ the function defined as the integral of $\phi_{F}(x, y, z, t)$ over the whole range of $y$

$$
q_{F}(x, z, t)=\int_{-\infty}^{+\infty} \phi_{F}(x, y, z, t) d y
$$

Making the realistic assumptions that $\lim _{y \rightarrow \pm \infty} \phi_{F}(x, y, z, t)=0$ and that partial derivatives of $\phi_{F}$ with respect to $x, y$ and $z$ tend also to zero when $y$ tends to infinity, we obtain, after integrating (6) with respect to $y$, the following two dimensional PDE

$$
\begin{aligned}
0= & \frac{\partial q_{F}}{\partial t}+\int\left(r_{d}(t)-f_{d}(0, t)\right) \phi_{F} d y+\frac{\partial}{\partial x}\left(\int\left(r_{d}(t)-r_{f}(t)\right) S(t) \phi_{F} d y\right) \\
& +\frac{\partial\left[\left(\theta_{f}(t)-\alpha_{f}(t) r_{f}(t)-\rho_{f S} \sigma_{f}(t) \sigma(t, S(t))\right) q_{F}\right]}{\partial z}-\frac{1}{2} \frac{\partial^{2}\left[\sigma^{2}(t, S(t)) S^{2}(t) q_{F}\right]}{\partial x^{2}} \\
& -\frac{1}{2} \frac{\partial^{2} \sigma_{f}^{2}(t) q_{F}}{\partial z^{2}}-\frac{\partial^{2} \sigma(t, S(t)) S(t) \sigma_{f}(t) \rho_{S f} q_{F}}{\partial x \partial z} .
\end{aligned}
$$

Finally, we define $p_{F}(x, t)$ as the integral of $q_{F}(x, z, t)$ over the whole range of $z$

$$
p_{F}(x, t)=\int_{-\infty}^{+\infty} q_{F}(x, z, t) d z=\int_{-\infty}^{+\infty} \int_{-\infty}^{+\infty} \phi_{F}(x, y, z, t) d y d z
$$


Assuming that $\lim _{z \rightarrow \pm \infty} q_{F}(x, z, t)=0$ and that partial derivatives of $q_{F}$ with respect to $x$ and $z$ tend also to zero when $z$ tends to the infinity, then integrating (8) with respect to $z$ reduces the PDE (8) into the following one-dimensional PDE

$$
\begin{aligned}
0= & \frac{\partial p_{F}}{\partial t}+\iint\left(r_{d}(t)-f_{d}(0, t)\right) \phi_{F} d y d z+\frac{\partial}{\partial x}\left(\iint\left(r_{d}(t)-r_{f}(t)\right) S(t) \phi_{F} d y d z\right) \\
& -\frac{1}{2} \frac{\partial^{2}\left[\sigma^{2}(t, S(t)) S^{2}(t) p_{F}\right]}{\partial x^{2}}
\end{aligned}
$$

\subsection{The local volatility derivation}

In this section we outline how to derive the expression of the local volatility function associated to the three-factor model with local volatility by differentiating the expression of a European call price $(C(K, t))$ with respect to its strike $K$ and its maturity $t$. In order to simplify our calculations, we will work with the forward call price $\widetilde{C}(K, t)$ defined by

$$
\widetilde{C}(K, t)=\frac{C(K, t)}{P_{d}(0, t)}=\mathbf{E}^{Q_{t}}\left[(S(t)-K)^{+}\right]=\iiint_{K}^{+\infty}(x-K) \phi_{F}(x, y, z, t) d x d y d z .
$$

Differentiating equation (11) twice with respect to $K$, we obtain

$$
\begin{aligned}
\frac{\partial \widetilde{C}(K, t)}{\partial K} & =\iiint_{K}^{+\infty}-\phi_{F}(x, y, z, t) d x d y d z=-\mathbf{E}^{Q_{t}}\left[\mathbf{1}_{\{S(t)>K\}}\right], \\
\frac{\partial^{2} \widetilde{C}(K, t)}{\partial K^{2}} & =\iint \phi_{F}(K, y, z, t) d y d z \equiv p_{F}(K, t) .
\end{aligned}
$$

Differentiating equation (11) with respect to the maturity and using equation (10) leads to

$$
\begin{aligned}
\frac{\partial \widetilde{C}(K, t)}{\partial t}= & \iiint_{K}^{+\infty}(S(t)-K) \frac{\partial \phi_{F}(x, y, z, t)}{\partial t} d x d y d z \\
= & \int_{K}^{+\infty}(S(t)-K) \frac{\partial p_{F}}{\partial t} d x \\
= & \int_{K}^{+\infty}(S(t)-K)\left\{-\iint\left(r_{d}(t)-f_{d}(0, t)\right) \phi_{F} d y d z\right. \\
& \left.-\frac{\partial}{\partial x}\left(\iint\left(r_{d}(t)-r_{f}(t)\right) S(t) \phi_{F} d y d z\right)+\frac{1}{2} \frac{\partial^{2}\left[\sigma^{2}(t, S(t)) S^{2}(t) p_{F}\right]}{\partial x^{2}}\right\} d x
\end{aligned}
$$

Integrating by parts several times and using equation (13) we get 


$$
\begin{aligned}
\frac{\partial \widetilde{C}(K, t)}{\partial t}= & f_{d}(0, t) \widetilde{C}(K, t)+\iiint_{K}^{+\infty}\left[r_{d}(t) K-r_{f}(t) S(t)\right] \phi_{F}(x, y, z, t) d x d y d z \\
& +\frac{1}{2}(\sigma(t, K) K)^{2} \frac{\partial^{2} \widetilde{C}(K, t)}{\partial K^{2}} \\
= & f_{d}(0, t) \widetilde{C}(K, t)+\mathbf{E}^{Q_{t}}\left[\left(r_{d}(t) K-r_{f}(t) S(t)\right) \mathbf{1}_{\{S(t)>K\}}\right] \\
& +\frac{1}{2}(\sigma(t, K) K)^{2} \frac{\partial^{2} \widetilde{C}(K, t)}{\partial K^{2}} .
\end{aligned}
$$

This leads to the following expression for the local volatility surface in terms of the forward call prices $\widetilde{C}(K, t)$

$$
\sigma^{2}(t, K)=\frac{\frac{\partial \widetilde{C}(K, t)}{\partial t}-f_{d}(0, t) \widetilde{C}(K, t)-\mathbf{E}^{Q_{t}}\left[\left(r_{d}(t) K-r_{f}(t) S(t)\right) \mathbf{1}_{\{S(t)>K\}}\right]}{\frac{1}{2} K^{2} \frac{\partial^{2} \widetilde{C}(K, t)}{\partial K^{2}}} .
$$

The (partial) derivatives of the forward call price with respect to the maturity and the strike can be easily calculated

$$
\begin{aligned}
\frac{\partial \widetilde{C}(K, t)}{\partial t} & =\frac{\partial\left[\frac{C(K, t)}{P_{d}(0, t)}\right]}{\partial t}=\frac{\partial C(K, t)}{\partial t} \frac{1}{P_{d}(0, t)}+f_{d}(0, t) \widetilde{C}(K, t) \\
\frac{\partial^{2} \widetilde{C}(K, t)}{\partial K^{2}} & =\frac{\partial^{2}\left[\frac{C(K, t)}{P_{d}(0, t)}\right]}{\partial K^{2}}=\frac{1}{P_{d}(0, t)} \frac{\partial^{2} C(K, t)}{\partial K^{2}} .
\end{aligned}
$$

Substituting these expressions $(16)$ and $(17)$ into $(15)$, we obtain the expression of the local volatility $\sigma^{2}(t, K)$ in terms of call prices $C(K, t)$

$$
\sigma^{2}(t, K)=\frac{\frac{\partial C(K, t)}{\partial t}-P_{d}(0, t) \mathbf{E}^{Q t}\left[\left(r_{d}(t) K-r_{f}(t) S(t)\right) \mathbf{1}_{\{S(t)>K\}}\right]}{\frac{1}{2} K^{2} \frac{\partial^{2} C(K, t)}{\partial K^{2}}} .
$$

Unfortunately, this extension of the Dupire formula is not easily applicable for calibration over the market since there seems no immediate way to link the expectation term with European option prices or other liquid products. However, we present in section 4 four different methods to calibrate this local volatility function.

Finally, we underline the fact that when assuming deterministic interest rates, equation (18) reduces to the simple Dupire formula corresponding to the one factor Gaussian case. This formula can easily be derived by a similar reasoning as above but in a one factor framework.

$$
\sigma^{2}(t, K)=\frac{\frac{\partial C(K, t)}{\partial t}+\left(r_{d}(t)-r_{f}(t)\right) K \frac{\partial C(K, t)}{\partial K}+r_{f}(t) C(K, t)}{\frac{1}{2} K^{2} \frac{\partial^{2} C(K, t)}{\partial K^{2}}} .
$$


Remark 1 The market often quotes options in terms of implied volatilities $\sigma_{\text {imp }}$ instead of option prices. Consequently, it is more convenient to express the local volatility in terms of implied volatilities than option prices. As the implied volatility of an option with price $C(K, T)$ is defined through the Black-Scholes formula $\left(C^{m k t}(K, T)=C^{B S}\left(K, T, \sigma_{i m p}\right)\right)$, the derivatives of call prices in equation (19) can be computed through the chain rule, and this leads to the following equation (see [Wilmott, 2006])

$$
\sigma^{2}(t, K)=\frac{\sigma_{i m p}^{2}+2 t \sigma_{i m p} \frac{\partial \sigma_{i m p}}{\partial t}+2\left(r_{d}(0)-r_{f}(0)\right) K t \sigma_{i m p} \frac{\partial \sigma_{i m p}}{\partial K}}{\left(1+K d_{+} \sqrt{t} \frac{\partial \sigma_{i m p}}{\partial K}\right)^{2}+K^{2} t \sigma_{i m p}\left(\frac{\partial^{2} \sigma_{i m p}}{\partial K^{2}}-d_{+}\left(\frac{\partial \sigma_{i m p}}{\partial K}\right)^{2} \sqrt{t}\right)} .
$$

Using the same approach, the local volatility expression (18) can be written in terms of implied volatilities $\sigma_{i m p}$,

$\sigma^{2}(t, K)=\sigma_{i m p} \frac{e^{-r_{f}(0) t} S(0)\left\{\mathcal{N}^{\prime}\left(d_{+}\right)\left(\sigma_{i m p}+2 t \frac{\partial \sigma_{i m p}}{\partial t}\right)-2 \sqrt{t} r_{f}(0) \mathcal{N}\left(d_{+}\right)\right\}+2 \sqrt{t}\left\{r_{d}(0) K e^{-r_{d}(0) t} \mathcal{N}\left(d_{-}\right)+E\right\}}{e^{-r_{f}(0) t} S(0) \mathcal{N}^{\prime}\left(d_{+}\right)\left\{\left(1+K d_{+} \sqrt{t} \frac{\partial \sigma_{i m p}}{\partial K}\right)^{2}+K^{2} t \sigma_{i m p}\left(\frac{\partial^{2} \sigma_{i m p}}{\partial K^{2}}-d_{+}\left(\frac{\partial \sigma_{i m p}}{\partial K}\right)^{2} \sqrt{t}\right)\right\}}$

where

$$
\begin{gathered}
E=P_{d}(0, t) \mathbf{E}^{Q_{t}}\left[\left(r_{d}(t) K-r_{f}(t) S(t)\right) 1_{\{S(t)>K\}}\right], \\
d_{ \pm}=\frac{\log \frac{S(0)}{K}+\left(r_{d}(0)-r_{f}(0) \pm \frac{\sigma_{i m p}^{2}}{2}\right) t}{\sigma_{i m p} \sqrt{t}} \\
\mathcal{N}(x)=\int_{-\infty}^{x} \frac{1}{\sqrt{2 \pi}} e^{\frac{-z^{2}}{2}} d z \\
\mathcal{N}^{\prime}(x)=\frac{1}{\sqrt{2 \pi}} e^{\frac{-x^{2}}{2}} .
\end{gathered}
$$

\section{Calibrating the Local Volatility}

Before using a model to price any derivatives, it is usual to calibrate it on the vanilla market which means that you should be able to price vanilla options with your model such that the resulting implied volatilities match the market-quoted ones. More precisely you have to determine all parameters present in the different stochastic processes which define the model in such a way that all European option prices derived in the model are as consistent as possible with the corresponding market ones.

The calibration procedure for the three-factor model with local volatility can be decomposed in three steps: (i) Parameters present in the Hull-White one-factor dynamics for the domestic and foreign interest rates, $\theta_{d}(t), \alpha_{d}(t), \sigma_{d}(t), \theta_{f}(t), \alpha_{f}(t), \sigma_{f}(t)$, are chosen to match European swaption / cap-floors 
values in their respective currencies. Methods for doing so are well developed in the literature (see for example Brigo and Mercurio, 2006]). (ii) The three correlation coefficients of the model, $\rho_{S d}, \rho_{S f}$ and $\rho_{d f}$ are usually estimated from historical data. (iii) After these two steps, the calibration problem consists in finding the local volatility function of the spot FX rate which is consistent with an implied volatility surface. In Piterbarg, 2006, Piterbarg derives an approximative formula for European call prices in the three-factor model where the local volatility function for the spot FX rate is a parametric function of the form $\sigma(t, S(t))=\nu(t)\left(\frac{S(t)}{L(t)}\right)^{\beta(t)-1}$, where $\nu(t)$ is the relative volatility function, $\beta(t)$ is a time-dependent elasticity of variance $(\mathrm{CEV})$ and $L(t)$ is a time-dependent scaling constant. The calibration procedure of Piterbarg consists in determining the functions $\nu(t)$ and $\beta(t)$ such that when pricing any European call with his approximative call valuation formula, the price he gets is as close as possible to the market call price. Next sections will be devoted to other calibration methods for the local volatility function $\sigma(t, S(t))$ based on the exact expression of this function in a three-factor context.

\subsection{Numerical approaches}

\subsubsection{A Monte Carlo approach}

In this section we present a first calibration method for the local volatility expression (18) derived in section 3. In this approach, the expectation, $\mathbf{E}^{Q_{T}}\left[\left(r_{d}(T) K-r_{f}(T) S(T)\right) \mathbf{1}_{\{S(T)>K\}}\right]$ is approximated by using Monte Carlo simulations up to a fixed time $t=T$. To calculate numerically this expectation we have to simulate the FX spot rate $S(t)$ and both the domestic and foreign interest rates up to time $T$ starting from the initial market prices $S(0), r_{d}(0)$ and $r_{f}(0)$ respectively. Since the expectation is expressed under the measure $Q_{T}$, we have to use the dynamics of $S(t), r_{d}(t)$ and $r_{f}(t)$ under this last measure,

$$
\left\{\begin{array}{l}
d S(t)=\left[r_{d}(t)-r_{f}(t)-\sigma(t, S(t)) \sigma_{d}(t) b_{d}(t, T) \rho_{S d}\right] S(t) d t+\sigma(t, S(t)) S(t) d W_{S}^{T F}(t) \\
d r_{d}(t)=\left[\theta_{d}(t)-\alpha_{d} r_{d}(t)-\sigma_{d}^{2}(t) b_{d}(t, T)\right] d t+\sigma_{d}(t) d W_{d}^{T F}(t) \\
d r_{f}(t)=\left[\theta_{f}(t)-\alpha_{f} r_{f}(t)-\rho_{f S} \sigma_{f}(t) b_{f}(t, T) \sigma(t, S(t))-\sigma_{d}(t) b_{d}(t, T) \sigma_{f}(t) b_{f}(t, T)\right] \rho_{d f} d t+\sigma_{f}(t) d W_{f}^{T F}(t)
\end{array}\right.
$$

where $b_{j}(t, T)=\frac{1}{\alpha_{j}}\left(1-e^{-\alpha_{j}(T-t)}\right), j=d, f$ (assuming that $\alpha_{j}$ are constant).

The idea of the Monte Carlo method is to simulate $n$ times (i.e. $n$ scenarios) the stochastic variables $S(t), r_{d}(t)$ and $r_{f}(t)$ up to time $T$, by using for example Euler discretisations. The expectation is approximated by:

$$
\mathbf{E}^{Q_{T}}\left[\left(r_{d}(T) K-r_{f}(T) S(T)\right) \mathbf{1}_{\{S(T)>K\}}\right] \cong \frac{1}{n} \sum_{i=1}^{n}\left(r_{d}^{i}(T) K-r_{f}^{i}(T) S^{i}(T)\right) \mathbf{1}_{\left\{S^{i}(T)>K\right\}}
$$

where $i$ corresponds to the $i^{t h}$-scenario $i=1, \ldots, n$.

As we have to know the local volatility function up to time $T$ to simulate the path for $S(t)$ and $r_{f}(t)$, the only way to work is forward in time. To begin, we have to determine the local volatility function at the first time step $T=T_{1}$ for all strike $K$. At this first step we assume that the initial local volatility is equal to the deterministic local volatility given by equation (19). Note that this 
local volatility is directly obtained by using market data (see equation (20). More precisely, by this choice, we assume that for a "small time period", interest rates are constant and in this case, the local volatility expression (18) reduces to (19). Knowing that local volatility function we can simulate

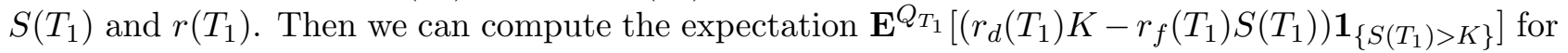
all $K$ by using:

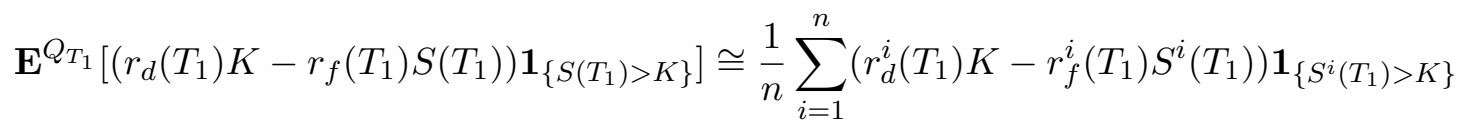

This allows us to get the local volatility expression at time $T_{1}, \sigma^{2}\left(T_{1}, K\right)$, for all strike $K$.

Following the same procedure we can easily calibrate the local volatility at time $T_{2}$ by using the local volatility obtained at time $T_{1}$ and also the simulated path until time $T_{1}$. Following this procedure we are able to generate the local volatility expression up to a final date $T=T_{k}$.

\subsubsection{A PDE approach}

The strategy is to solve the forward equation (6) forwards one step at a time, starting with a local volatility $\sigma(0, S(0))$ at time $t_{0}=0$. At the first time step $t_{1}=t_{0}+\Delta t$, we can generate the forward joint transition densities $\phi_{F}\left(x, y, z, t_{0}+\Delta t\right)$ by solving the forward PDE using the initial condition $\phi_{F}\left(x, y, z, t_{0}\right)=\delta\left(x-x_{0}, y-y_{0}, z-z_{0}\right)$ at time $t_{0}=0$. Knowing the forward joint transition densities we can calculate the expectation in 18 namely $\mathbf{E}^{Q_{t}}\left[\left(r_{d}(t) K-r_{f}(t) S(t)\right) \mathbf{1}_{\{S(t)>K\}}\right]$ at this point $t_{1}$. This allows us to calculate from equation (18) the function $\sigma\left(t_{1}, S\left(t_{1}\right)\right)$ at this time step. Following this procedure through time, we generate simultaneously both the expectation in $(18)$ and the local volatility function $\sigma(t, S(t))$. At each time step we generate also all domestic forward joint transition densities $\phi_{F}\left(S(t), r_{d}(t), r_{f}(t), t\right)$ by solving the forward PDE (6).

\subsection{Comparison between local volatility with and without stochastic interest rates}

In this section we present a way to calibrate the extension of the local volatility function derived in section 3 in a three-factor framework by using the local volatility showing up in the simple one-factor Gaussian model.

Assuming deterministic interest rates, the FX spot follows the diffusion equation

$$
d S(t)=\left(r_{d}(t)-r_{f}(t)\right) S(t) d t+\sigma_{1 f}(t, S(t)) S(t) d W_{S}^{D R N}(t)
$$

where the local volatility function denoted by $\sigma_{1 f}$ is given by equation 19

$$
\sigma_{1 f}^{2}(t, K)=\frac{\frac{\partial C(K, t)}{\partial t}+K\left(f_{d}(0, t)-f_{f}(0, t)\right) \frac{\partial C(K, t)}{\partial K}+f_{f}(0, t) C(K, t)}{\frac{1}{2} K^{2} \frac{\partial^{2} C(K, t)}{\partial K^{2}}} .
$$

However, if we consider the three-factor model with stochastic interest rates, the local volatility function is given by equation 18

\footnotetext{
${ }^{1}$ in the case of deterministic interest rates $f_{d}(0, t)=r_{d}(t)$ and $f_{f}(0, t)=r_{f}(t)$
} 


$$
\sigma_{3 f}^{2}(t, K)=\frac{\frac{\partial C(K, t)}{\partial t}-P_{d}(0, t) \mathbf{E}^{Q t}\left[\left(K r_{d}(t)-r_{f}(t) S(t)\right) \mathbf{1}_{\{S(t)>K\}}\right]}{\frac{1}{2} K^{2} \frac{\partial^{2} C(K, t)}{\partial K^{2}}} .
$$

Using the fact that under the $T$-forward measure for $T \geq t$, we have: $\mathbf{E}^{Q_{T}}\left[r_{d}(T) \mid \mathcal{F}_{t}\right]=f_{d}(t, T)$ and $\mathbf{E}^{Q_{T}}\left[r_{f}(T) \mid \mathcal{F}_{t}\right]=f_{f}(t, T)$ we notice that

$$
\begin{aligned}
\mathbf{E}^{Q_{t}}\left[r_{d}(t) \mathbf{1}_{\{S(t)>K\}}\right] & =\mathbf{E}^{Q_{t}}\left[r_{d}(t)\right] \mathbf{E}^{Q_{t}}\left[\mathbf{1}_{\{S(t)>K\}}\right]+\operatorname{Cov}^{Q_{t}}\left[r_{d}(t), \mathbf{1}_{\{S(t)>K\}}\right] \\
& =f_{d}(0, t)\left(-\frac{1}{P_{d}(0, t)} \frac{\partial C(t, K)}{\partial K}\right)+\operatorname{Cov}^{Q_{t}}\left[r_{d}(t), \mathbf{1}_{\{S(t)>K\}}\right]
\end{aligned}
$$

where $\operatorname{Cov}^{Q_{t}}(X, Y)$ represents the covariance between two stochastic variables $\mathrm{X}$ and $\mathrm{Y}$ with dynamics expressed in the $t$-forward measure $Q_{t}$. We also have

$$
\begin{aligned}
\mathbf{E}^{Q_{t}}\left[r_{f}(t) S(t) \mathbf{1}_{\{S(t)>K\}}\right]= & \mathbf{E}^{Q_{t}}\left[r_{f}(t)(S(t)-K) \mathbf{1}_{\{S(t)>K\}}\right]+\mathbf{E}^{Q_{t}}\left[r_{f}(t) K \mathbf{1}_{\{S(t)>K\}}\right] \\
= & \mathbf{E}^{Q_{t}}\left[r_{f}(t)\right] \mathbf{E}^{Q_{t}}\left[(S(t)-K)^{+}\right]+\mathbf{C o v}^{Q_{t}}\left[r_{f}(t),(S(t)-K)^{+}\right] \\
& +K\left(\mathbf{E}^{Q_{t}}\left[r_{f}(t)\right] \mathbf{E}^{Q_{t}}\left[\mathbf{1}_{\{S(t)>K\}}\right]+\operatorname{Cov}^{Q_{t}}\left[r_{f}(t), \mathbf{1}_{\{S(t)>K\}}\right]\right) \\
= & f_{f}(0, t) \frac{C(t, K)}{P_{d}(0, t)}+\mathbf{C o v}^{Q_{t}}\left[r_{f}(t),(S(t)-K)^{+}\right] \\
& +K\left(f_{f}(0, t)\left(-\frac{1}{P_{d}(0, t)} \frac{\partial C(t, K)}{\partial K}\right)+\operatorname{Cov}^{Q_{t}}\left[r_{f}(t), \mathbf{1}_{\{S(t)>K\}}\right]\right) .
\end{aligned}
$$

Substituting expressions (27) and (28) in equation (26), one finds the following interesting relation between the simple Dupire formula (25) and its extension (26)

$\sigma_{3 f}^{2}(t, K)-\sigma_{1 f}^{2}(t, K)=\frac{K P_{d}(0, t)\left\{\operatorname{Cov}^{Q_{t}}\left[r_{f}(t)-r_{d}(t), \mathbf{1}_{\{S(t)>K\}}\right]+\frac{1}{K} \operatorname{Cov}^{Q_{t}}\left[r_{f}(t),(S(t)-K)^{+}\right]\right\}}{\frac{1}{2} K^{2} \frac{\partial^{2} C}{\partial K^{2}}}$.

Assuming that quantities $\operatorname{Cov}^{Q t}\left[r_{f}(t),(S(t)-K)^{+}\right]$and $\operatorname{Cov}^{Q t}\left[r_{f}(t)-r_{d}(t), \mathbf{1}_{\{S(t)>K\}}\right]$ are extractable from the market, equation (29) shows the corrections to make to the tractable Dupire local volatility surface in order to obtain the local volatility surface which takes into account the effects of both domestic and foreign stochastic interest rates.

\subsection{Calibrating the local volatility by mimicking stochastic volatility models}

In this section we consider the three factor model with a stochastic volatility for the spot FX rate and we show how to connect this model to the one where the spot FX rate volatility is a local volatility. More precisely, we sketch the derivation of an expectation relationship between local volatilities and future instantaneous spot FX rate volatilities. 
Consider the following domestic risk neutral dynamics for the spot FX rate

$$
d S(t)=\left(r_{d}(t)-r_{f}(t)\right) S(t) d t+\gamma(t, \nu(t)) S(t) d W_{S}^{D R N}(t)
$$

where $\nu(t)$ is a stochastic variable which provides the stochastic perturbation for the spot FX rate volatility. Common choices for the function $\gamma(t, \nu(t))$ are $\nu(t), \exp (\sqrt{\nu(t)})$ and $\sqrt{\nu(t)}$. The stochastic variable $\nu(t)$ is generally modelled by a Cox-Ingersoll-Ross (CIR) process (mean-reversion in the drift and a volatility dependent of $\sqrt{\nu(t)}$ as for example the Heston model Heston, 1993]) or in the first case by a Ornstein-Uhlenbeck process (OU) (mean-reversion in the drift and a volatility independent from $\nu(t)$ as for example the Schöbel and Zhu [Schöbel and Zhu, 1999] stochastic volatility model) which allows for negative values of $\nu(t)$.

Applying Tanaka's formula to the convex but non-differentiable function $e^{-\int_{0}^{t} r_{d}(s) d s}(S(t)-K)^{+}$ leads to

$$
\begin{aligned}
e^{-\int_{0}^{t} r_{d}(s) d s}(S(t)-K)^{+}= & (S(0)-K)^{+}-\int_{0}^{t} r_{d}(u) e^{-\int_{0}^{u} r_{d}(s) d s}(S(u)-K)^{+} d u \\
& +\int_{0}^{t} e^{-\int_{0}^{u} r_{d}(s) d s} \mathbf{1}_{\{S(u)>K\}} d S_{u}+\frac{1}{2} \int_{0}^{t} e^{-\int_{0}^{u} r_{d}(s) d s} d L_{u}^{K}(S)
\end{aligned}
$$

where $L_{u}^{K}(S)$ is the local time of $S$. Since $S$ is a continuous semimartingale, then almost-surely (see Revuz and Yor, 2001])

$$
L_{t}^{K}(S)=\lim _{\epsilon \downarrow 0} \frac{1}{\epsilon} \int_{0}^{t} \mathbf{1}_{[K, K+\epsilon]}(S(s)) d<S, S>_{s} .
$$

Using the domestic risk neutral diffusion for the spot FX rate 30 , one obtains

$$
\begin{aligned}
e^{-\int_{0}^{t} r_{d}(s) d s}(S(t)-K)^{+}= & (S(0)-K)^{+}+K \int_{0}^{t} r_{d}(u) e^{-\int_{0}^{u} r_{d}(s) d s} \mathbf{1}_{\{S(u)>K\}} d u \\
& -\int_{0}^{t} e^{-\int_{0}^{u} r_{d}(s) d s} \mathbf{1}_{\{S(u)>K\}} r_{f}(u) S(u) d u \\
& +\int_{0}^{t} e^{-\int_{0}^{u} r_{d}(s) d s} \mathbf{1}_{\{S(u)>K\}} \gamma(u, \nu(u)) S(u) d W_{S}^{D R N}(u) \\
& +\frac{1}{2} \int_{0}^{t} e^{-\int_{0}^{u} r_{d}(s) d s} d L_{u}^{K}(S) .
\end{aligned}
$$

Assuming that the function $e^{-\int_{0}^{u} r_{d}(s) d s} \mathbf{1}_{\{S(u)>K\}} \gamma(u, \nu(u)) S(u)$ is a member of the class $\mathcal{H}^{2}$, namely the measurable and adapted functions $f$ such that $\mathbf{E}^{Q_{d}}\left[\int_{0}^{t} f^{2}(s) d s\right]<\infty$, we get when taking the domestic risk neutral expectation of each side

$$
\begin{aligned}
\mathbf{E}^{Q_{d}}\left[e^{-\int_{0}^{t} r_{d}(s) d s}(S(t)-K)^{+}\right]= & \mathbf{E}^{Q_{d}}\left[(S(0)-K)^{+}\right]+K \int_{0}^{t} \mathbf{E}^{Q_{d}}\left[r_{d}(u) e^{-\int_{0}^{u} r_{d}(s) d s} \mathbf{1}_{\{S(u)>K\}}\right] d u \\
& -\int_{0}^{t} \mathbf{E}^{Q_{d}}\left[e^{-\int_{0}^{u} r_{d}(s) d s} \mathbf{1}_{\{S(u)>K\}} r_{f}(u) S(u)\right] d u \\
& +\int_{0}^{t} \frac{1}{2} \mathbf{E}^{Q_{d}}\left[e^{-\int_{0}^{u} r_{d}(s) d s} d L_{u}^{K}(S)\right] .
\end{aligned}
$$


Differentiating this equation leads to

$$
\begin{aligned}
d C(K, t)= & K \mathbf{E}^{Q_{d}}\left[r_{d}(t) e^{-\int_{0}^{t} r_{d}(s) d s} \mathbf{1}_{\{S(t)>K\}}\right] d t-\mathbf{E}^{Q_{d}}\left[e^{-\int_{0}^{t} r_{d}(s) d s} \mathbf{1}_{\{S(t)>K\}} r_{f}(t) S(t)\right] d t \\
& +\frac{1}{2} \mathbf{E}^{Q_{d}}\left[e^{-\int_{0}^{t} r_{d}(s) d s} d L_{t}^{K}(S)\right] .
\end{aligned}
$$

Using characterization (31) for the local time with $d<S, S>_{t}=\gamma^{2}(t, \nu(t)) S^{2}(t) d t$, we obtain

$$
\begin{aligned}
d C(K, t)= & K \mathbf{E}^{Q_{d}}\left[r_{d}(t) e^{-\int_{0}^{t} r_{d}(s) d s} \mathbf{1}_{\{S(t)>K\}}\right] d t-\mathbf{E}^{Q_{d}}\left[e^{-\int_{0}^{t} r_{d}(s) d s} \mathbf{1}_{\{S(t)>K\}} r_{f}(t) S(t)\right] d t \\
& +\frac{1}{2} \lim _{\epsilon \downarrow 0} \mathbf{E}^{Q_{d}}\left[\frac{1}{\epsilon} \mathbf{1}_{[K, K+\epsilon]}(S(t)) e^{-\int_{0}^{t} r_{d}(s) d s} \gamma^{2}(t, \nu(t)) S^{2}(t)\right] d t .
\end{aligned}
$$

Here, the last terms of the equation $(48)$ can be rewritten as

$$
\begin{aligned}
& \lim _{\epsilon \downarrow 0} \frac{1}{\epsilon} \mathbf{E}^{Q_{d}}\left[\mathbf{1}_{[K, K+\epsilon]}(S(t)) e^{-\int_{0}^{t} r_{d}(s) d s} \gamma^{2}(t, \nu(t)) S^{2}(t)\right] \\
& =\lim _{\epsilon \downarrow 0} \frac{1}{\epsilon} \mathbf{E}^{Q_{d}}\left[\mathbf{E}^{Q_{d}}\left[\gamma^{2}(t, \nu(t)) e^{-\int_{0}^{t} r_{d}(s) d s} \mid S(t)\right] \mathbf{1}_{[K, K+\epsilon]}(S(t)) S^{2}(t)\right] \\
& =\mathbf{E}^{Q_{d}}\left[\gamma^{2}(t, \nu(t)) e^{-\int_{0}^{t} r_{d}(s) d s} \mid S(t)=K\right] p_{d}(K, t) K^{2} \\
& =\frac{\mathbf{E}^{Q_{d}}\left[\gamma^{2}(t, \nu(t)) e^{-\int_{0}^{t} r_{d}(s) d s} \mid S(t)=K\right]}{\mathbf{E}^{Q_{d}}\left[e^{-\int_{0}^{t} r_{d}(s) d s} \mid S(t)=K\right]} \frac{\partial^{2} C(K, t)}{\partial K^{2}} K^{2} .
\end{aligned}
$$

Substituting equation (49) in (48), one obtains

$$
\frac{\mathbf{E}^{Q_{d}}\left[\gamma^{2}(t, \nu(t)) e^{-\int_{0}^{t} r_{d}(s) d s} \mid S(t)=K\right]}{\mathbf{E}^{Q_{d}}\left[e^{-\int_{0}^{t} r_{d}(s) d s} \mid S(t)=K\right]}=\frac{\frac{\partial C}{\partial t}-\mathbf{E}^{Q_{d}}\left[e^{-\int_{0}^{t} r_{d}(s) d s}\left(K r_{d}(t)-r_{f}(t) S(t)\right) \mathbf{1}_{\{S(t)>K\}}\right]}{\frac{1}{2} K^{2} \frac{\partial^{2} C}{\partial K^{2}}} .
$$

The right hand side of this equation equals the local volatility in the three-factor model where the expectation is expressed under the domestic risk-neutral measure. Therefore, if there exists a local volatility such that the one-dimensional probability distribution of the spot FX rate with the diffusion (1) is the same as the one of the spot FX rate with dynamics (30) for every time $t$, then this local volatility function has to satisfy the following equation

$$
\sigma^{2}(t, K)=\frac{\mathbf{E}^{Q_{d}}\left[\gamma^{2}(t, \nu(t)) e^{-\int_{0}^{t} r_{d}(s) d s} \mid S(t)=K\right]}{\mathbf{E}^{Q_{d}}\left[e^{-\int_{0}^{t} r_{d}(s) d s} \mid S(t)=K\right]} .
$$

Stochastic volatility models belong to the class of incomplete market models, and hence, we have to precise that this condition holds if we assume that the domestic risk neutral probability measure $Q_{d}$ used in both stochastic and local volatility framework are the same. This equation extends the result obtained by Dupire [Dupire, 2004] and Derman and Kani [Derman and Kani, 1998] to the case where domestic and foreign interest rates are stochastic. We notice that when we assume deterministic interest rates, equation (51) reduces exactly to their result 


$$
\sigma^{2}(t, K)=\mathbf{E}^{Q_{d}}\left[\gamma^{2}(t, \nu(t)) \mid S(t)=K\right] .
$$

Finally, we reduce equation (51) to a conditional expectation under the $t$-forward measure

$$
\sigma^{2}(t, K)=\mathbf{E}^{Q_{t}}\left[\gamma^{2}(t, \nu(t)) \mid S(t)=K\right] .
$$

The local volatility $\sigma^{2}(t, S(t)=K)$ is, therefore, the conditional expectation under the $t$-forward measure of the instantaneous spot FX rate volatility at the future time $t$, contingent on the spot FX rate level $S(t)$ being equal to $K$.

Equation (59) give us a new way to calibrate the local volatility function. A numerical approach is to begin with solving the forward PDE (6) by using for example finite differences or finite elements methods, to determine the forward probability density $\phi_{F}$ and afterwards using numerical integration methods in order to calculate the conditional expectation (59).

Conditional expectations are difficult to compute by using traditional Monte Carlo simulations since paths generated by the simulation will miss the event involved in the conditional expectation. However, Malliavin integration by parts allows to obtain different representations of such conditional expectation that can be compute efficiently by Monte Carlo simulations (see [Fournié et al., 2001]).

Remark 2 If we assume independence between the spot FX rate and its volatility, the local volatility function is given by $\sigma^{2}(T, K)=\mathbf{E}^{Q_{T}}\left[\gamma^{2}(T, \nu(T))\right]$. In some particular cases it is possible to derive closed-form solutions for this expectation. Consider for example the three-factor model with local volatility where domestic and foreign interest rates have Hull and White dynamics (see section 2) and we want to find the local volatility function by using the mimicking property (59) derived from a fourfactor model with the same interest rates dynamics and with Schöbel and Zhu dynamics for the FX spot volatility $(\nu(t))$,

$$
\left\{\begin{array}{l}
d S(t)=\left(r_{d}(t)-r_{f}(t)\right) S(t) d t+\nu(t) S(t) d W_{S}^{D R N}(t) \\
d r_{d}(t)=\left[\theta_{d}(t)-\alpha_{d} r_{d}(t)\right] d t+\sigma_{d} d W_{d}^{D R N}(t), \\
d r_{f}(t)=\left[\theta_{f}(t)-\alpha_{f} r_{f}(t)-\rho_{f S} \sigma_{f} \nu(t)\right] d t+\sigma_{f} d W_{f}^{D R N}(t), \\
d \nu(t)=k[\lambda-\nu(t)] d t+\xi d W_{\nu}^{D R N}(t)
\end{array}\right.
$$

Under the T-Forward measure, the Schöbel and Zhu dynamics (41) become

$$
d \nu(t)=k(\Lambda(t)-\nu(t)) d t+\xi d W_{\nu}^{T F}(t),
$$

where $\Lambda(t)=\lambda-\frac{\rho_{d \nu} \sigma_{d} b_{d}(t, T) \xi}{k}, b_{d}(t, T)=\frac{1}{\alpha_{d}}\left(1-e^{-\alpha_{d}(T-t)}\right)$

Integrating equation (42), we obtain, for each $t \leq T$

$$
\nu(T)=\nu(t) e^{-k(T-t)}+\int_{t}^{T} k \Lambda(u) e^{-k(T-u)} d u+\int_{t}^{T} \xi e^{-k(T-t)} d W_{\nu}^{T F}(u),
$$


so that $\nu(T)$ conditional on $\mathcal{F}_{t}$ is normally distributed with mean and variance given respectively by

$$
\begin{aligned}
\mathbf{E}^{Q_{T}}\left[\nu(T) \mid \mathcal{F}_{t}\right] & =\nu(t) e^{-k(T-t)}+\left(\lambda-\frac{\rho_{d \nu} \sigma_{d} \xi}{\alpha_{d} k}\right)\left(1-e^{-k(T-t)}\right)+\frac{\rho_{d \nu} \sigma_{d} \xi}{\left.\alpha_{d}\left(\alpha_{d}+k\right)\right)}\left(1-e^{-\left(\alpha_{d}+k\right)(T-t)}\right) \\
\operatorname{Var}^{Q_{T}}\left[\nu(T) \mid \mathcal{F}_{t}\right] & =\frac{\xi^{2}}{2 k}\left(1-e^{-2 k(T-t)}\right)
\end{aligned}
$$

Finally, the closed form solution for the local volatility function is given by

$$
\begin{aligned}
\sigma^{2}(T, K) & =\mathbf{E}^{Q_{T}}\left[\nu^{2}(T)\right]=\left(\mathbf{E}^{Q_{T}}[\nu(T)]\right)^{2}+\operatorname{Var}^{Q_{T}}[\nu(T)] \\
& =\left(\nu(t) e^{-k T}+\left(\lambda-\frac{\rho_{d \nu} \sigma_{d} \xi}{\alpha_{d} k}\right)\left(1-e^{-k T}\right)+\frac{\rho_{d \nu} \sigma_{d} \xi}{\left.\alpha_{d}\left(\alpha_{d}+k\right)\right)}\left(1-e^{-\left(\alpha_{d}+k\right) T}\right)\right)^{2}+\frac{\xi^{2}}{2 k}\left(1-e^{-2 k T}\right)
\end{aligned}
$$

\section{$5 \quad$ Hybrid volatility model}

In a simple local volatility model, the instantaneous volatility of the spot FX rate is a deterministic function of time and spot FX level. As a consequence, these models are suitable for pricing derivatives in situations where the spot FX volatility is strongly correlated to the spot FX market level itself. However, in the FX option's market, the volatility seems to exhibit some stochastic behavior especially in the long dated market. In this section we study an extension of the three-factor model with local volatility which incorporates stochastic behavior in the spot FX volatility without deleting the local volatility one.

In this section we consider a hybrid volatility model where the volatility of the spot FX rate is a combination of a stochastic and a local volatility function. More precisely, the volatility for the spot FX rate corresponds to a local volatility $\sigma_{L O C 2}(t, S(t))$ multiplied by a stochastic volatility $\gamma(t, \nu(t))$ where $\nu(t)$ is a stochastic variable. This gives the following four-factor model with local volatility

$$
\left\{\begin{array}{l}
d S(t)=\left(r_{d}(t)-r_{f}(t)\right) S(t) d t+\sigma_{L O C 2}(t, S(t)) \gamma(t, \nu(t)) S(t) d W_{S}^{D R N}(t) \\
d r_{d}(t)=\left[\theta_{d}(t)-\alpha_{d}(t) r_{d}(t)\right] d t+\sigma_{d}(t) d W_{d}^{D R N}(t) \\
d r_{f}(t)=\left[\theta_{f}(t)-\alpha_{f}(t) r_{f}(t)-\rho_{f S} \sigma_{f}(t) \sigma_{L O C 2}(t, S(t)) \gamma(t, \nu(t))\right] d t+\sigma_{f}(t) d W_{f}^{D R N}(t) \\
d \nu(t)=\alpha(t, \nu(t)) d t+\vartheta(t, \nu(t)) d W_{\nu}^{D R N}(t) .
\end{array}\right.
$$

We will show how to connect this model to the one where the spot FX rate volatility is a pure local volatility. In this case the volatility of the spot FX rate is modelled by a local volatility denoted by $\sigma_{L O C 1}(t, S(t))$

$$
\left\{\begin{array}{l}
d S(t)=\left(r_{d}(t)-r_{f}(t)\right) S(t) d t+\sigma_{L O C 1}(t, S(t)) S(t) d W_{S}^{D R N}(t) \\
d r_{d}(t)=\left[\theta_{d}(t)-\alpha_{d}(t) r_{d}(t)\right] d t+\sigma_{d}(t) d W_{d}^{D R N}(t) \\
d r_{f}(t)=\left[\theta_{f}(t)-\alpha_{f}(t) r_{f}(t)-\rho_{f S} \sigma_{f}(t) \sigma_{L O C 1}(t, S(t))\right] d t+\sigma_{f}(t) d W_{f}^{D R N}(t) .
\end{array}\right.
$$

Consider the domestic risk neutral dynamics for the spot FX rate in the Hybrid volatility model (equation(45)) 


$$
d S(t)=\left(r_{d}(t)-r_{f}(t)\right) S(t) d t+\sigma_{L O C 2}(t, S(t)) \gamma(t, \nu(t)) S(t) d W_{S}^{D R N}(t)
$$

where $\nu(t)$ is a stochastic variable which provides the stochastic perturbation for the spot FX rate volatility. Applying Tanaka's formula to the non-differentiable function $e^{-\int_{0}^{t} r_{d}(s) d s}(S(t)-K)^{+}$and following the same steps as in section 4.3 leads to

$$
\begin{aligned}
d C(K, t)= & K \mathbf{E}^{Q_{d}}\left[r_{d}(t) e^{-\int_{0}^{t} r_{d}(s) d s} \mathbf{1}_{\{S(t)>K\}}\right] d t-\mathbf{E}^{Q_{d}}\left[e^{-\int_{0}^{t} r_{d}(s) d s} \mathbf{1}_{\{S(t)>K\}} r_{f}(t) S(t)\right] d t \\
& +\frac{1}{2} \lim _{\epsilon \downarrow 0} \mathbf{E}^{Q_{d}}\left[\frac{1}{\epsilon} \mathbf{1}_{[K, K+\epsilon]}(S(t)) e^{-\int_{0}^{t} r_{d}(s) d s} \sigma_{L O C 2}^{2}(t, S(t)) \gamma^{2}(t, \nu(t)) S^{2}(t)\right] d t .
\end{aligned}
$$

Here, the last terms of the equation 48 can be rewritten as

$$
\begin{aligned}
& \lim _{\epsilon \downarrow 0} \frac{1}{\epsilon} \mathbf{E}^{Q_{d}}\left[\mathbf{1}_{[K, K+\epsilon]}(S(t)) e^{-\int_{0}^{t} r_{d}(s) d s} \sigma_{L O C 2}^{2}(t, S(t)) \gamma^{2}(t, \nu(t)) S^{2}(t)\right] \\
& =\lim _{\epsilon \downarrow 0} \frac{1}{\epsilon} \mathbf{E}^{Q_{d}}\left[\mathbf{E}^{Q_{d}}\left[\gamma^{2}(t, \nu(t)) e^{-\int_{0}^{t} r_{d}(s) d s} \mid S(t)\right] \mathbf{1}_{[K, K+\epsilon]}(S(t)) \sigma_{L O C 2}^{2}(t, S(t)) S^{2}(t)\right] \\
& =\mathbf{E}^{Q_{d}}\left[\gamma^{2}(t, \nu(t)) e^{-\int_{0}^{t} r_{d}(s) d s} \mid S(t)=K\right] p_{d}(K, t) \sigma_{L O C 2}^{2}(t, K) K^{2} \\
& =\frac{\mathbf{E}^{Q_{d}}\left[\gamma^{2}(t, \nu(t)) e^{-\int_{0}^{t} r_{d}(s) d s} \mid S(t)=K\right]}{\mathbf{E}^{Q_{d}}\left[e^{-\int_{0}^{t} r_{d}(s) d s} \mid S(t)=K\right]} \frac{\partial^{2} C(K, t)}{\partial K^{2}} \sigma_{L O C 2}^{2}(t, K) K^{2} .
\end{aligned}
$$

Substituting equation $(49)$ in $(48)$, one obtains

$\sigma_{L O C 2}^{2}(t, K) \frac{\mathbf{E}^{Q_{d}}\left[\gamma^{2}(t, \nu(t)) e^{-\int_{0}^{t} r_{d}(s) d s} \mid S(t)=K\right]}{\mathbf{E}^{Q_{d}}\left[e^{-\int_{0}^{t} r_{d}(s) d s} \mid S(t)=K\right]}=\frac{\frac{\partial C}{\partial t}-\mathbf{E}^{Q_{d}}\left[e^{-\int_{0}^{t} r_{d}(s) d s}\left(K r_{d}(t)-r_{f}(t) S(t)\right) \mathbf{1}_{\{S(t)>K\}}\right]}{\frac{1}{2} K^{2} \frac{\partial^{2} C}{\partial K^{2}}}$.

The right hand side of this equation equals the local volatility in the three-factor model where the expectation is expressed under the domestic risk-neutral measure. Therefore, if there exists a local volatility such that the one-dimensional probability distribution of the spot FX rate with the diffusion (46) is the same as the one of the spot FX rate with dynamics 477 for every time $t$, then this local volatility function has to satisfy the following equation

$$
\begin{aligned}
\sigma_{L O C 1}^{2}(t, K) & =\sigma_{L O C 2}^{2}(t, K) \frac{\mathbf{E}^{Q_{d}}\left[\gamma^{2}(t, \nu(t)) e^{-\int_{0}^{t} r_{d}(s) d s} \mid S(t)=K\right]}{\mathbf{E}^{Q_{d}}\left[e^{-\int_{0}^{t} r_{d}(s) d s} \mid S(t)=K\right]} \\
& =\sigma_{L O C 2}^{2}(t, K) \mathbf{E}^{Q_{t}}\left[\gamma^{2}(t, \nu(t)) \mid S(t)=K\right] .
\end{aligned}
$$

Hybrid volatility models belong also to the class of incomplete market models, and hence, we assume that the domestic risk neutral probability measure $Q_{d}$ used in both hybrid and pure local volatility framework are the same. This equation extends the result obtained by Madan Madan et al. 2007 to the case where domestic and foreign interest rates are stochastic. We notice that when we assume deterministic interest rates, equation (51) reduces exactly to their result 


$$
\sigma_{L O C 1}^{2}(t, K)=\sigma_{L O C 2}^{2}(t, K) \mathbf{E}^{Q_{d}}\left[\gamma^{2}(t, \nu(t)) \mid S(t)=K\right] .
$$

The local volatility $\sigma_{L O C 2}^{2}(t, K)$ is, therefore, given by the local volatility $\sigma_{L O C 1}^{2}(t, K)$ divided by the conditional expectation under the $t$-forward measure of the instantaneous spot FX rate volatility at the future time $t$, contingent on the spot FX rate level $S(t)$ being equal to $K$.

$$
\sigma_{L O C 2}^{2}(t, K)=\frac{\sigma_{L O C 1}^{2}(t, K)}{\mathbf{E}^{Q_{t}}\left[\gamma^{2}(t, \nu(t)) \mid S(t)=K\right]}
$$

Equation (54) give us a way to calibrate the local volatility function $\sigma_{L O C 2}(t, K)$ knowing the local volatility function $\sigma_{L O C 1}(t, K)$. A numerical approach is to begin with solving the forward PDE (6) by using for example finite differences or finite elements methods, to determine the forward probability density $\phi_{F}$ and afterwards using numerical integration methods in order to calculate the conditional expectation (54) (see subsection 5.1). As mentioned in section 4.3, an other approach is to use Malliavin integration by parts to obtain different representations of the conditional expectation that can be computed efficiently by Monte Carlo simulations (see [Fournié et al. 2001]).

Remark 3 If we assume independence between the spot FX rate and its volatility, the local volatility

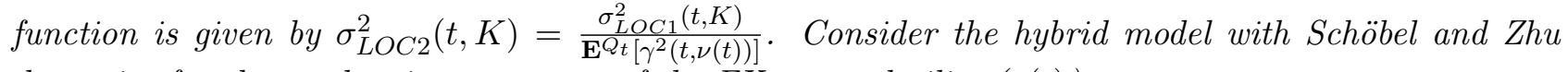
dynamics for the stochastic component of the $F X$ spot volatility $(\nu(t))$,

$$
\left\{\begin{array}{l}
d S(t)=\left(r_{d}(t)-r_{f}(t)\right) S(t) d t+\sigma_{L O C 2}(t, S(t)) \nu(t) S(t) d W_{S}^{D R N}(t), \\
d r_{d}(t)=\left[\theta_{d}(t)-\alpha_{d} r_{d}(t)\right] d t+\sigma_{d} d W_{d}^{D R N}(t), \\
d r_{f}(t)=\left[\theta_{f}(t)-\alpha_{f} r_{f}(t)-\rho_{f S} \sigma_{f} \nu(t)\right] d t+\sigma_{f} d W_{f}^{D R N}(t), \\
d \nu(t)=k[\lambda-\nu(t)] d t+\xi d W_{\nu}^{D R N}(t)
\end{array}\right.
$$

In this particular case we have a closed form solution for the local volatility function $\sigma_{L O C 2}$, (knowing the local volatility function $\sigma_{L O C 1}$ ) given by

$$
\sigma_{L O C 2}^{2}(t, K)=\frac{\sigma_{L O C 1}^{2}(t, K)}{\left(\nu(t) e^{-k T}+\left(\lambda-\frac{\rho_{d \nu} \sigma_{d} \xi}{\alpha_{d} k}\right)\left(1-e^{-k T}\right)+\frac{\rho_{d \nu} \sigma_{d} \xi}{\left.\alpha_{d}\left(\alpha_{d}+k\right)\right)}\left(1-e^{-\left(\alpha_{d}+k\right) T}\right)\right)^{2}+\frac{\xi^{2}}{2 k}\left(1-e^{-2 k T}\right)} .
$$

In this section we obtained a link between the pure local volatility model (46) and the hybrid volatility model (45) such that the one-dimensional marginal distributions of $S_{t}$ is the same for every $t$ in both models. Using this result for the calibration of the local volatility $\sigma_{L O C 2}(t, S(t)$ ) (see section 5.1) will guarantee that prices of Vanilla options will be the same in both models since these products are fully determined by the conditional probability of the spot FX rate at the maturity time $T$ conditional to the actual spot FX rate value. However, when we price exotic and path dependent options where payoff depends on intermediate spot values, pricing requires the product of all conditional probabilities. By using this link, we have never imposed that these probabilities are the same. 
As a consequence, prices coming from the hybrid volatility model (45) are different from those coming from the pure local volatility one (46) as well as the pure stochastic volatility one (30) in the case of exotic derivatives. The advantage of the hybrid volatility model is that we have a combination of a local and a stochastic volatility effect in the volatility of the spot FX rate which is more realistic. Consequently, hybrid volatility models are generally more consistent with the exotic option's market after being calibrated with respect to the vanilla market (see [Lipton and McGhee, 2002]).

\subsection{Calibration}

In this subsection we are interested in the calibration of the local volatility $\sigma_{L O C 2}(t, S(t))$ of the fourfactor hybrid volatility model knowing the local volatility $\sigma_{L O C 1}(t, S(t))$ associated to the three-factor model that we have studied in the first part of this paper. In [Madan et al., 2007], the authors obtain a fast and exact calibration of vanilla options in the case of equity options with deterministic interest rates. Based on results obtained in this section, we extend this calibration procedure to the case of FX derivatives in the settings of stochastic interest rates.

Remember that parameters present in the Hull-White one-factor dynamics for the domestic and foreign interest rates, $\theta_{d}(t), \alpha_{d}(t), \sigma_{d}(t), \theta_{f}(t), \alpha_{f}(t), \sigma_{f}(t)$, are chosen to match European swaption / cap-floors values in their respective currencies. The four correlation coefficients of the model, $\rho_{S d}, \rho_{S f}$, $\rho_{d f}$ and $\rho_{S \nu}$ are deduced from historical data. In this hybrid volatility model one also has to calibrate the process for $\nu(t)$. The volatility of the variance $\vartheta(t, \nu(t))$ can be deduced from historical time series with for example daily realized volatilities. If one uses a CIR process to model the stochastic variable $\vartheta(t, \nu(t))$, then it is of the form $\vartheta(t, \nu(t))=\beta(t) \sqrt{\nu(t)}$, while if one models it by using an OU process, $\vartheta(t, \nu(t))$ is independent of $\nu(t)$ and given by $\vartheta(t, \nu(t))=\beta(t)$. Usually, practitioners use a mean-reverting form for the drift of the variance, $\alpha(t, \nu(t))=\lambda(t)-\kappa(t) \nu(t)$. One also has to calibrate the local volatility $\sigma_{L O C 1}(t, S(t))$ associated to the three-factor model with a local volatility only. This calibration can be realized using methods developed in section 4. Once all these parameters have been determined, one is able to calibrate the local volatility function $\sigma_{L O C 2}(t, S(t))$ associated to the hybrid volatility model by using equation (54), namely

$$
\sigma_{L O C 2}^{2}(t, K)=\frac{\sigma_{L O C 1}^{2}(t, K)}{\mathbf{E}^{Q_{t}}\left[\gamma^{2}(t, \nu(t)) \mid S(t)=K\right]}
$$

where the conditional expectation is by definition given by

$$
\begin{aligned}
& \mathbf{E}^{Q_{t}}\left[\gamma^{2}(t, \nu(t)) \mid S(t)=K\right] \\
& =\frac{\int_{0}^{\infty} \gamma^{2}(t, \nu(t)) \phi_{F}\left(S(t)=K, r_{d}(t), r_{f}(t), \nu(t), t\right) d \nu}{\int_{0}^{\infty} \phi_{F}\left(S(t)=K, r_{d}(t), r_{f}(t), \nu(t), t\right) d \nu}
\end{aligned}
$$

The $t$-forward joint density $\phi_{F}\left(S(t), r_{d}(t), r_{f}(t), \nu(t), t\right)$ satisfies a four-dimensional Kolmogorov forward equation that can be derived using the same method as in section 3.1 .

The strategy is to solve this forward equation forwards one step at a time, starting with a local volatility $\sigma_{L O C 2}(0, S(0))$ equal to $\sigma_{L O C 1}(0, S(0)) / \gamma(0, \nu(0))$ at time $t_{0}=0$, since the volatility $\nu(0)$ is known and we have $\mathbf{E}^{Q_{t}}[\gamma(0, \nu(0)) \mid S(0)=K]=\gamma(0, \nu(0))$. At the time step $t_{1}=t_{0}+\Delta t$ we can 
generate the forward joint transition densities $\phi_{F}\left(x, y, z, \nu, t_{0}+\Delta t\right)$ by solving the forward PDE using the initial condition $\phi_{F}\left(x, y, z, \nu, t_{0}\right)=\delta\left(x-x_{0}, y-y_{0}, z-z_{0}, \nu-\nu_{0}\right)$ at time $t_{0}=0$. Knowing the $t$-forward joint transition densities we can calculate each integral in equation (61) to determine the function $\mathbf{E}^{Q_{t}}\left[\gamma\left(t_{1}, \nu\left(t_{1}\right)\right) \mid S\left(t_{1}\right)=K\right]$ at this point. This allows us to calculate from equation 60 the function $\sigma_{L O C 2}\left(t_{1}, S\left(t_{1}\right)\right)$ at this time step. Following this procedure through time, we generate simultaneously both the conditional expectation $\mathbf{E}^{Q_{t}}[\gamma(t, \nu(t)) \mid S(t)=K]$ and the local volatility function $\sigma_{L O C 2}(t, S(t))$. At each time step we generate also all forward joint transition densities $\phi_{F}\left(S(t), r_{d}(t), r_{f}(t), \nu(t), t\right)$ by solving the forward PDE.

\section{Conclusion}

We have derived the local volatility expression of the spot FX rate in a stochastic interest rates framework. Therefore this model is very promising in pricing and hedging long-dated FX derivatives which are more and more traded in the FX option's market. We have proposed four different approaches for the calibration of this local volatility function. First we have proposed two numerical approaches based on Monte Carlo methods and numerical resolution of a PDE, respectively. The third one is based on the difference between the tractable local volatility surface that exists in the context of deterministic interest rates and our generalized one. The last method consists in generating the local volatility surface from a stochastic volatility. More precisely, we have obtained an explicit link between the local volatility and the stochastic volatility and this in a stochastic interest rates framework. This method has the advantage to give a smoother and more stable local volatility surface than when it is built from market available option prices.

Afterwards, we have considered an extension of the previous model which allows the volatility of the spot FX rate to have local and stochastic behavior. Indeed, we have studied a hybrid volatility model, where the volatility of the spot FX rate is the product of a local volatility and a stochastic volatility. We have obtained results useful for the calibration of this new local volatility with respect to the stochastic volatility and the local volatility studied in the first part of the paper.

The three-factor model with stochastic volatility and its extension will be useful in the pricing and risk management of long-dated FX derivatives for which it is especially important to consider the risk of both domestic/foreign interest rates and the risk linked to the FX spot volatility. Future studies will include numerical tests of the calibration methods on the FX market and comparison between their speed and appropriateness to fit the market implied volatility surface. The impact of stochastic interest rates and hedging performance of these models are also left for future research.

\section{References}

R. Ahlip. Foreign exchange options under stochastic volatility and stochastic interest rates. International Journal of Theoretical and Applied Finance, 11:277-294, 2008.

J. Andreasen. Closed form pricing of FX options under stochastic rates and volatility. In Global Derivatives Conference, ICBI, May 2006.

A. Antonov, M. Arneguy, and N. Audet. Markovian projection to a displaced volatility Heston model. Working paper, 2008. Available at http://ssrn.com/abstract=1106223. 
F. Bossens, G. Rayee, N.S. Skantzos, and G. Deelstra. Vanna-Volga methods applied to FX derivatives: from theory to market practice. International Journal of Theoretical and Applied Finance, 13(8): 1293-1324, 2010.

D. Brigo and F. Mercurio. Interest Rate Models - Theory and Practice: With Smile, Inflation and Credit. Springer-Verlag, 2nd edition, 2006.

E. Derman and I. Kani. Riding on a Smile. Risk, pages 32-39, 1994.

E. Derman and I. Kani. Stochastic implied trees: Arbitrage pricing with stochastic term and strike structure of volatility. International Journal of Theoretical and Applied Finance, 1:61-110, 1998.

B. Dupire. Pricing with a Smile. Risk, pages 18-20, 1994.

B. Dupire. A Unified Theory of Volatility in Derivatives Pricing: The Classic Collection, chapter 6, pages 185-196. (Ed. P. Carr) Risk Books, 2004.

E. Fournié, J. M. Lasry, J. Lebuchoux, and P. L. Lions. Applications of malliavin calculus to MonteCarlo methods in finance. ii. Finance and Stochastics, 5(2):201-236, 2001.

L.A. Grzelak and C.W. Oosterlee. On Cross-Currency Models with Stochastic Volatility and Correlated Interest Rates. Working paper, Delft University of Technology, 2010. Available at http://papers.ssrn.com/sol3/papers.cfm?abstract_id=1618684.

S.L. Heston. A closed-form solution for options with stochastic volatility with applications to bond and currency options. Rev Fin Studies, 6:327-343, 1993.

J. Hull and A. White. One Factor Interest Rate Models and the Valuation of Interest Rate Derivative Securities. Journal of Financial and Quantitative Analysis, 28:235-254, 1993.

A. Lipton. The vol smile problem. Risk Magazine, 15:61-65, 2002.

A. Lipton and W. McGhee. Universal Barriers. Risk Magazine, 15:81-85, 2002.

D. Madan, M. Qian Qian, and Y. Ren. Calibrating and pricing with embedded local volatility models. Risk, 2007.

M. Overhaus, A. Bermudez, H. Buehler, A. Ferraris, C. Jordinson, and A. Lamnouar. Equity Hybrid Derivatives. Wiley Finance, 2006.

V. Piterbarg. Smiling hybrids. Risk, pages 66-71, 2006.

D. Revuz and M. Yor. Continuous Martingales and Brownian Motion. Springer-Verlag, 2001.

R. Schöbel and J. Zhu. Stochastic volatility with an Ornstein Uhlenbeck process: An extension. European Finance Review, 4:23-46, 1999.

W. Schoutens, E. Simons, and J. Tistaert. A Perfect Calibration! Now What? Wilmott Magazine, 2004.

J. Sippel and S. Ohkoshi. All power to PRDC notes. Risk Magazine, 15, 2002. 
D. Tavella, A. Giese, and D. Vermeiren. Hybrid Stochastic Volatility Calibration. The Best of Wilmott, $2: 221-228,2006$.

A. van Haastrecht, R. Lord, A. Pelsser, and D. Schrager. Pricing Long-Maturity Equity and FX Derivatives with Stochastic Interest Rates and Stochastic Volatility. Insurance: Mathematics and Economics, 45(3):436-448, 2009.

A. van Haastrecht and A. Pelsser. Generic Pricing of FX, Inflation and Stock Options Under Stochastic Interest Rates and Stochastic Volatility. Working paper (forthcoming in quantitative finance ), 2009. Available at http://papers.ssrn.com/sol3/papers.cfm?abstract_id=1197262.

P. Wilmott. Paul Wilmott on Quantitative Finance. John Wiley and Sons Ltd, 2nd edition, 2006. 